\title{
Pemphigus foliaceus IgG causes dissociation of desmoglein 1-containing junctions without blocking desmoglein 1 transinteraction
}

\author{
Jens Waschke, ${ }^{1}$ Paola Bruggeman, ${ }^{1}$ Werner Baumgartner, ${ }^{1}$ \\ Detlef Zillikens, ${ }^{2}$ and Detlev Drenckhahn'1
}

${ }^{1}$ Institute of Anatomy and Cell Biology, University of Würzburg, Würzburg, Germany. ${ }^{2}$ Department of Dermatology, University of Lübeck, Lübeck, Germany.

\begin{abstract}
Autoantibodies against the epidermal desmosomal cadherins desmoglein 1 (Dsg1) and Dsg3 have been shown to cause severe to lethal skin blistering clinically defined as pemphigus foliaceus (PF) and pemphigus vulgaris (PV). It is unknown whether antibody-induced dissociation of keratinocytes is caused by direct inhibition of Dsg1 transinteraction or by secondary cellular responses. Here we show in an in vitro system that IgGs purified from PF patient sera caused cellular dissociation of cultured human keratinocytes as well as significant release of Dsg1-coated microbeads attached to Dsg-containing sites on the keratinocyte cellular surface. However, cell dissociation and bead release induced by PF-IgGs was not caused by direct steric hindrance of Dsg1 transinteraction, as demonstrated by single molecule atomic force measurements and by laser trapping of surface-bound Dsg1-coated microbeads. Rather, our experiments strongly indicate that PF-IgG-mediated dissociation events must involve autoantibody-triggered cellular signaling pathways, resulting in destabilization of Dsg1-based adhesive sites and desmosomes.
\end{abstract}

\section{Introduction}

It is well established that skin blistering in patients suffering from pemphigus vulgaris (PV) and pemphigus foliaceus (PF) is caused by formation of antibodies against the desmosomal cadherin family members desmoglein 1 (Dsg1) and Dsg3, respectively (1-4). Dsgs are linked to the keratinocyte intermediate filament cytoskeleton by several adaptor proteins located in the desmosomal plaque, including plakoglobin $(5,6)$. It has been proposed that antibody-induced steric hindrance of Dsg transcellular binding (transinteraction) is the major pathogenic mechanism responsible for cellular dissociation and pemphigus development $(7,8)$. However, other mechanisms, including antibody-induced activation of extracellular proteolysis, phosphorylation of Dsgs, and activation of protein kinase $\mathrm{C}$ followed by plakoglobin dislocation and subsequent depletion of Dsgs from desmosomes, appear to be important for pemphigus pathogenesis (9-13). To address this question of direct inhibitory action of antibodies on desmosomal adhesion versus antibody-induced intracellular signaling pathways involved in cellular dissociation, we used single molecule-based micromechanical approaches, i.e., laser tweezers and atomic force microscopy (AFM). These approaches, which have been used in our laboratory to characterize binding properties and regulatory mechanisms of vascular endothelial cadherin (VE-cadherin) and neuronal cadherin (N-cadherin) (14-19), allowed us, for what we believe is the first time, to discriminate between direct and indirect effects of antibodies on Dsg-based intercellular adhesion.

Nonstandard abbreviations used: AFM, atomic force microscopy; Dsc, desmocollin; Dsg, desmoglein; F-actin, filamentous actin; Fab, antigen-binding fragment; $\mathrm{HaCaT}$, immortalized human keratinocyte cell line; N-cadherin, neuronal cadherin; NHS, $\mathrm{N}$-hydroxy-succimide; PEG, polyethylene glycol; PF, pemphigus foliaceus; PV, pemphigus vulgaris; RT, room temperature; VE-cadherin, vascular endothelial cadherin. Conflict of interest: The authors have declared that no conflict of interest exists.
In the present study, we provide evidence that PF-IgGs do not directly inhibit Dsg1-mediated adhesion by steric hindrance but require cell-dependent signaling mechanisms.

PF-IgGs at concentrations that induced cellular dissociation in monolayers of cultured immortalized human keratinocyte cell line $(\mathrm{HaCaT})$ keratinocytes also resulted in release of Dsg1-coated beads bound to Dsg-containing sites on the cell surface. In contrast, when beads were preincubated with PF-IgGs before settlement on $\mathrm{HaCaT}$ cells, binding to the cell surface was not inhibited.

\section{Results}

Effect of PF-IgGs on buman keratinocytes (HaCaT). IgG fractions from $P F$ patients as well as a monoclonal mouse antibody directed against the extracellular domain of Dsg1 detected the recombinant Dsg1-Fc fusion protein in dot blot analysis (Figure 1). Dot blot analysis of lysates of confluent $\mathrm{HaCaT}$ cells demonstrated the presence of Dsg1 as well as of Dsg3 (Figure 1). Dsg1 was hardly detectable 3 days after plating and increased over time until strong expression was detected at 7 days after plating, the time when experiments were performed. A mouse monoclonal antibody to Dsg3 was used for immunostaining of desmosomes in cultured $\mathrm{HaCaT}$ monolayers. In order to reveal autoantibodyinduced dissociation of keratinocytes at the light microscope level, we looked for a staining protocol that allowed visualization of even small intercellular gaps. Keratinocytes were strongly labeled by antibodies to cytokeratins, with the exception of the cell periphery where staining was weak (not shown). However, Alexa-phalloidin, a specific label for filamentous actin (F-actin), created strong labeling of the entire cortical cytoplasm and allowed detection of even very small intercellular gaps.

In control monolayers, Dsg3 and F-actin were strongly enriched along the cell periphery (Figure 2, A-C). Individual desmosomes were not revealed under these conditions. Moreover, Dsg3 was localized in cytoplasmic compartments, probably reflecting sites of synthesis, transport, and recycling (20). The overall distribu- 


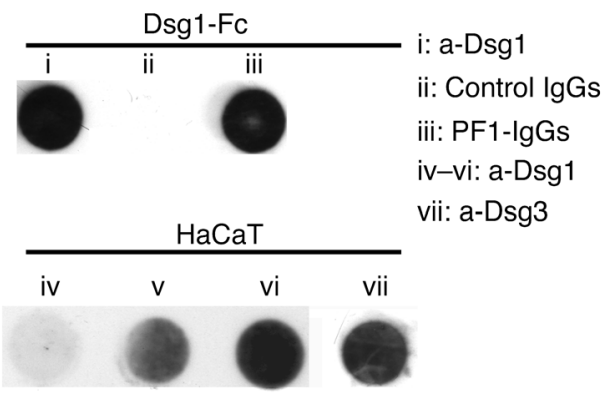

Figure 1

Characterization of PF-IgGs and HaCaT cells. Dot blot immunodetection demonstrating that the mouse monoclonal antibody directed against Dsg1 (i) as well as PF1-IgGs (iii) but not control IgGs (ii) detected the recombinant fusion protein consisting of the complete extracellular domain of Dsg1 and the Fc-portion of human IgGs (Dsg1-Fc). Both Dsg1 and Dsg3 were detected in HaCaT cell lysates by the monoclonal anti-Dsg1 (a-Dsg1) antibody (iv-vi) and the monoclonal a-Dsg3 antibody (vii), respectively. Contents of Dsg1 constantly increased from 3 days (iv) and 5 days (v) up to 7 days after plating (vi), the time when monolayers were used for experiments. The data shown is for 1 representative experiment out of 3 .

tion of F-actin and Dsg3 remained unchanged by incubation of $\mathrm{HaCaT}$ cells with control IgGs for 24 hours $(35 \mu \mathrm{g} / \mathrm{ml}$ ) (Figure 2, D-F). In contrast, when $\mathrm{HaCaT}$ cells were incubated with PF1-IgGs $(35 \mu \mathrm{g} / \mathrm{ml}$ total $\mathrm{IgG})$, intercellular gaps appeared between many cells of the monolayers (Figure $2 \mathrm{G}$ ) accompanied by profound changes in cell shape. Gap formation could be observed as early as after 2 hours of incubation with PF-IgGs (not shown), but effects were more pronounced after 24 hours of incubation. Under these conditions, different effects on the actin cytoskeleton were observed. F-actin had largely disappeared from cell borders (Figure 2, G and M). The cytoplasmatic actin filament system displayed formation of numerous stress fibers (Figure 2G), aggregates of short actin filaments, or a complete loss of cytoplasmatic actin (Figures 2M and 3C). Overall immunoreactivity for Dsg3 was reduced in cells displaying gap formation but was still present in small cellular processes interconnecting neighboring cells (arrowheads in Figure 2H). To investigate whether cell dissociation was caused by Dsg1-specific antibodies from the PF-IgG fractions, we performed immunoabsorption experiments. Immunoabsorption using beads coated with recombinant Dsg1-Fc abolished both gap formation and changes in cellular shape as documented for PF2-IgGs (Figure 2, J-L). In control experiments using beads coated with recombinant VE-cadherin$\mathrm{Fc}$ (which is not expressed in keratinocytes), the cell-dissociating activity of PF-IgGs remained unchanged (Figure 2, M-O), indicating that Dsg1-specific antibodies from the PF-IgG fractions were responsible for these effects. In contrast to PF-IgG, the monoclonal antibody (1:50, 24 hours) directed against the extracellular domain of Dsg1 did not induce cell dissociation (Figure 2, P-R) although it inhibited Dsg1-mediated homophilic binding as revealed by laser tweezer assay and AFM (see below) under the same conditions.

Next, we tested the effect of PF-IgGs on distribution of Dsg1. Under control conditions, Dsg1 was distributed along cell borders when $\mathrm{HaCaT}$ cells were grown to full confluence (Figure 3A). However, following treatment with PF-IgG, Dsg1 immunostaining was largely lost in cell groups in which gap formation and cell dissociation were detected by F-actin staining (Figure 3, B and C, arrows). On the other hand, immunostaining for Dsg3 was clearly less strongly affected.

Characterization of bead-to-cell junctions. For characterization of bead-to-cell junctions by immunofluorescence, we used Dsg1coated latex-sulfate beads rather than Dynabeads because the latter displayed strong autofluorescence whereas the former are nonfluorescent. After settlement on the surface of $\mathrm{HaCaT}$ cells for 30 minutes, most beads had recruited endogenous Dsg1, Dsg3, and plakoglobin to the bead-to-cell junctions (Figure 4, A-D), as shown by halo-like accumulation of immunofluorescence specific for these proteins in the immediate vicinity of the beads (arrows in Figure 4, B and D). Beads coated with Fc fragments only (used as controls) did not induce formation of such beadto-cell contacts (Figure 4, E and F). With respect to staining for Dsg1, it has to be mentioned that the Dsg1 antibody used for staining is directed against the extracellular domain of Dsg1 and thus cannot discriminate between cellular Dsg1 and recombinant Dsg1-Fc bound to the bead surface. However, as seen in Figure 4B, the amount of Dsg1-Fc bound to the bead surface was in most experiments too low to create any significant immunosignal. Beads not inducing immunopositive bead-to-cell contacts were in the range of roughly $10-30 \%$, which is close to the value of loosely attached beads determined by laser tweezer experiments $(20 \%)$. To further characterize the structure of bead-to-cell contacts, monolayers were processed for transmission and scanning electron microscopy. During the rather harsh conditions of fixation, dehydration, and embedding/critical point drying, only the tightly (specifically) bound beads remained attached to the cell surface. Cell-to-bead junctions were found at the tip of fingerlike processes between the cell surface and adhering microbeads (arrow in Figure 5). These processes closely mimicked the desmosome-bearing processes between neighboring cells (arrowhead). Phagocytosis of beads was not observed under these conditions and also did not occur during the subsequent incubation period with PF-IgGs (30-60 minutes).

PF-IgGs reduced adhesion of Dsg1-coated microbeads to the surface of $\mathrm{HaCaT}$ cells. The effect of PF-IgG fractions on Dsg1 binding was studied by laser tweezer experiments. For this purpose, Dsg1-coated beads were allowed to settle on the cell surface for 30 minutes before their binding was tested by separating forces of the laser beam. Under these control conditions, about 80\% of Dsg1-coated beads were tightly bound to the cell surface. Incubation periods longer than 30 minutes did not change the overall outcome of the assay. Therefore, all studies were performed with the 30-minute protocol. When EGTA ( $5 \mathrm{mM}, 30$ minutes) was added to the culture medium, the number of bound beads dropped to $27 \% \pm 1 \%$ of control values (Figure 6), documenting that Dsg1-mediated adhesion is strongly $\mathrm{Ca}^{2+}$-dependent $(n=6)$. To demonstrate that bead binding was specifically mediated by Dsg 1 and to rule out the possibility that loss of bead binding following incubation with EGTA was caused by secondary effects, we performed experiments with the mouse monoclonal antibody directed against the extracellular domain of Dsg1. At antibody concentrations of 1:50, the immunolabeling signal of dot blots of recombinant Dsg1 displayed intensities similar to the signal obtained with PF-IgGs (Figure 1). AntiDsg1 reduced the number of bound Dsg1-coated beads to $\mathrm{HaCaT}$ cells to $27 \% \pm 7 \%$ of control values and thus to the same extent as EGTA. In the presence of control IgGs $(35 \mu \mathrm{g} / \mathrm{ml})$, the number of 

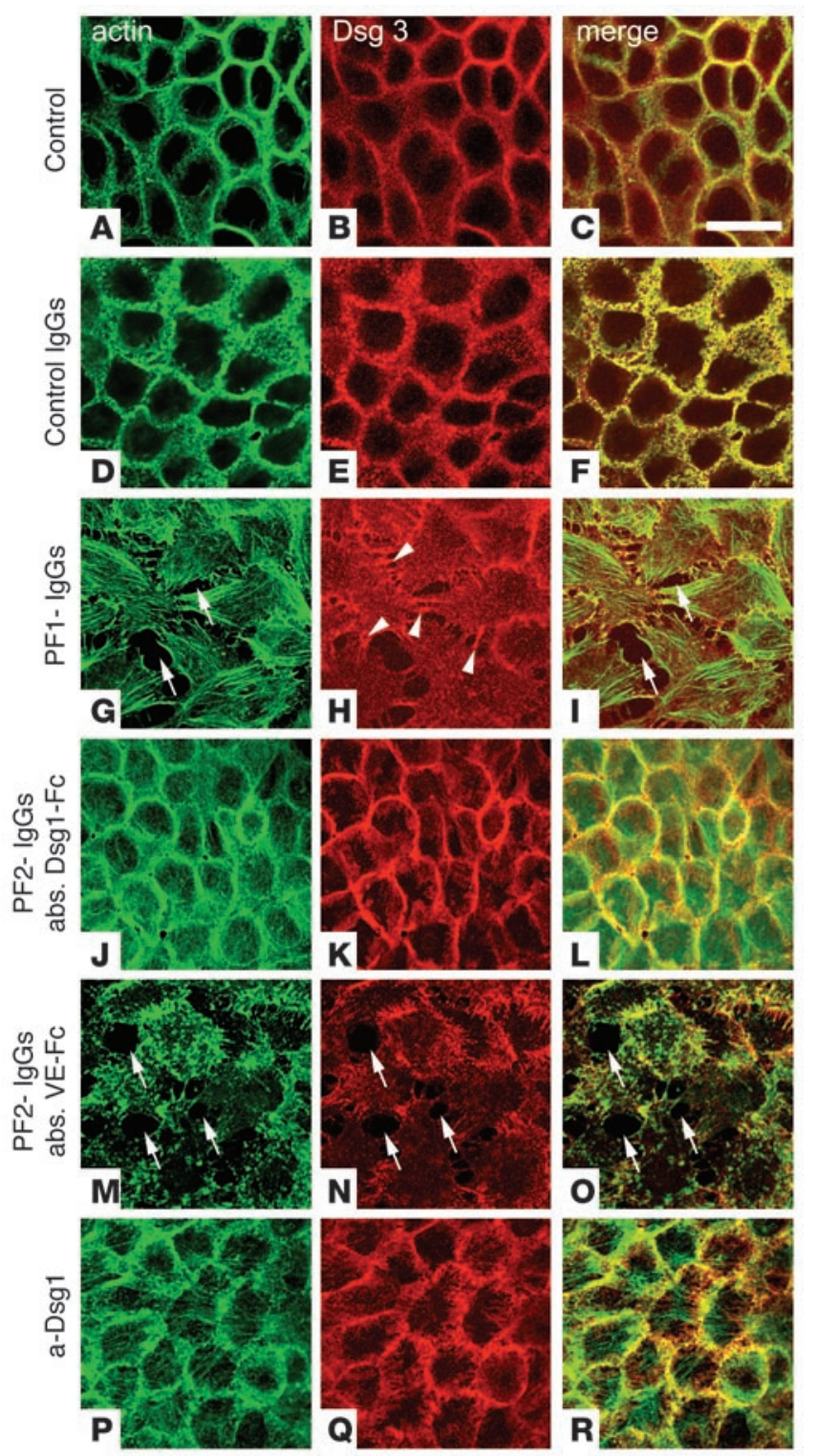

beads bound to the cell surface was unaffected $(n=6)$. However, when $\mathrm{HaCaT}$ cells with surface-bound beads were postincubated with PF-IgG fractions (PF1- and PF2-IgGs, $35 \mu \mathrm{g} / \mathrm{ml}, 30$ minutes), the number of bound beads dropped significantly to $51 \% \pm 2 \%$ and $28 \% \pm 4 \%$ of control values, respectively $(n=6)$. When PF2IgGs were depleted of Dsg1 autoantibodies by immunoabsorption with Dsg1-coated beads (see above), the PF2-IgG-induced loss of bead binding was completely abolished (Figure 6), indicating that Dsg1-specific antibodies from the PF-IgG fraction were responsible for these effects. This conclusion was confirmed by control experiments using VE-cadherin-coated beads for immunoabsorption. No significant reduction of PF2-IgG-induced loss of Dsg1mediated binding was observed under these conditions ( $42 \% \pm 4 \%$ of control values, $n=5$ each).

To investigate whether PF-IgGs inhibit Dsg1-mediated adhesion directly or act indirectly by complex cellular responses, we performed preincubation studies in which either HaCaT cells or Dsg1-coated beads were preincubated with PF-IgGs before beads

\section{Figure 2}

PF-IgG-induced cell dissociation in HaCaT monolayers. HaCaT cells double-stained for F-actin using Alexa-phalloidin (A, D, G, J, M, and P) and Dsg3 (B, E, H, K, N, and $\mathbf{Q}$ ). In controls, F-actin and the desmosomal cadherin Dsg3 were distributed along cell junctions (A-C). Control IgGs (35 $\mu \mathrm{g} / \mathrm{ml}, 24$ hours) did not affect distribution of Dsg3 (E and F). In contrast, PF1-IgGs (35 $\mu \mathrm{g} / \mathrm{ml}, 24$ hours) induced intercellular gaps (arrows) best seen in the Alexa-phalloidin stain for F-actin ( $G$ and $\mathbf{I})$. Note that Dsg3 is still present in cell processes spanning gaps (arrowheads in $\mathbf{H}$ ). Following immunoabsorption of PF-IgGs by Dsg1-Fc-coated beads, PF2-lgGs ( $35 \mu \mathrm{g} / \mathrm{ml}, 24$ hours) had no effect (J-L) whereas large gaps (arrows) were induced by incubation with PF2-IgGs when control absorption was performed with beads coated with VE-cadherin-Fc (VE-Fc) (M-O), indicating requirement of autoantibodies specific for Dsg1 for cell dissociation. Note that the inhibitory monoclonal antibody directed against the extracellular domain of Dsg1 did not induce gaps (P-R). Scale bar: $40 \mu \mathrm{m}$ for all panels $(n=5)$. abs., immunoabsorption.

were allowed to settle on the cell surface. When HaCaT cells $(35 \mu \mathrm{g} / \mathrm{ml})$ were preincubated for 30 minutes with PF1-IgGs before beads were applied after removal of PF-IgG-containing culture medium, binding of Dsg1-beads was reduced to $33 \% \pm 8 \%$ of control levels and thus was not significantly different from experiments in which PF-IgGs were applied subsequently to binding of Dsg1-coated beads $(n=5)$. However, when instead Dsg1coated beads were preincubated with PF1-IgGs (35 $\mu \mathrm{g} / \mathrm{ml}, 30 \mathrm{~min}-$ utes) in vitro before settlement on $\mathrm{HaCaT}$ cells, $93 \% \pm 3 \%$ of beads compared with controls bound to the cell surface, indicating that PF-IgGs did not inhibit Dsg1-mediated binding to HaCaT cells $(n=5)$. In contrast, the monoclonal anti-Dsg1 reduced bead binding to $29 \% \pm 2 \%$ when cells were preincubated with the antibody and also reduced the number of bound beads to $26 \% \pm 1 \%$ when Dsg1-coated beads were preincubated with the antibody, indicating that the mechanisms by which PF-IgGs and the monoclonal antibody affected Dsg1-mediated adhesion are profoundly different. Moreover, these experiments demonstrate that PF-IgG treatment of cultured keratinocytes reduced Dsg1-mediated adhesion not by steric hindrance of Dsg 1 transinteraction but by involved secondary cellular mechanisms.

PF-IgGs did not block transinteraction of single Dsg1 molecules in a cell-free system. To study the effects of PF-IgGs in a cell-free system where adhesion of Dsg1 molecules can be investigated in the absence of any cellular signaling mechanisms, we used single molecule atomic force microscopy (AFM). Force-distance cycles were performed to quantify binding activity between Dsg1-Fc molecules coupled to tip and plate of the AFM setup as described in detail elsewhere $(14,16,21)$. The general principle of determination of binding activity of transinteracting recombinant Dsg1 dimers is illustrated in Figure 7A. Dsg1 covalently attached to the tip of the cantilever and the surface of the plate were brought into interaction and separation by cyclic upward and downward movements of the tip at a frequency of $1-2 \mathrm{~Hz}$ (force-distance cycles). During downward movement (approach), the tip of the cantilever eventually hit the plate and, during further downward movement, was gradually deflected upwards until the end of the approach (defined as zero position of the cycle). During the following retrace movement, the cantilever bent back with the same linear force slope until the tip separated from the plate to reach the unbent neutral position. During further progression of the retrace movement, the cantilever remained in the neutral position if no interaction 


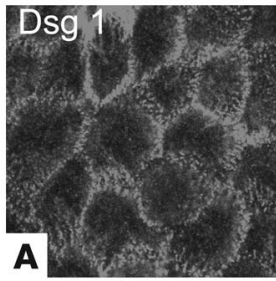

Control

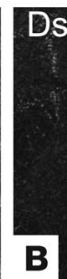

B

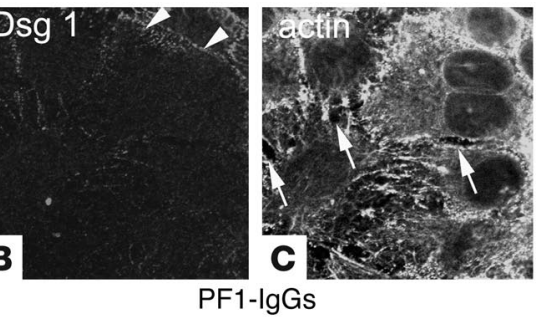

Figure 3

PF-IgG-induced cell dissociation is accompanied by loss of Dsg1 staining. After incubation of $\mathrm{HaCaT}$ cells with PF-IgGs $(35 \mu \mathrm{g} / \mathrm{ml}$, 24 hours), monolayers were double-stained for Dsg1 (B) and F-actin using Alexa-phalloidin (C). Intercellular gaps are indicated by arrows. Dsg1 is located along cell borders $(\mathbf{A})$ and becomes strongly reduced in response to PF-IgGs (arrowheads in B). Scale bar: $40 \mu \mathrm{m}$ for all panels $(n=5)$.

occurred between tip- and plate-bound Dsgs. Interaction between Dsg1 molecules proceeded throughout the entire period during which plate and tip were in contact. If interaction had taken place, the cantilever was pulled down below the neutral line until a critical force was reached (unbinding force) at which the Dsg1 bonds broke. Unbinding was followed by an abrupt jump of the cantilever to the neutral position. Unbinding events typically proceeded successively, creating saw tooth-like unbinding curves such as those shown in Figure 7A. Because Dsg1 was attached to the plate and tip by flexible polyethylene glycol (PEG) linkers, molecules could freely diffuse within the radius of the length of the linkers $(\sim 8 \mathrm{~nm})$, allowing them to undergo unimpaired encounter reactions (21). Approach-retrace cycles of $800 \mathrm{~nm} / \mathrm{s}$ and 0.1 seconds encounter time were performed in the presence and absence of antibodies. The total area between the force curve and the neutral line was taken as a measure for binding activity (Figure 7A) as outlined previously (14). Binding activity was normalized to experiments using a cantilever not labeled with Dsg1 to eliminate the contribution of unspecific interactions. Each point of the plot shown in Figure 7A represents the mean activity of at least 600 retrace cycles using one AFM tip (4 tips for each condition).

The unit unbinding force of a single binding event of 2 transinteracting Dsg1 molecules was measured as $37-68 \mathrm{pN}$ depending on the pulling velocity $(300 \mathrm{~nm} / \mathrm{s}-6000 \mathrm{~nm} / \mathrm{s})$, which is comparable to the unbinding force of other cadherins, such as VE-cadherin or $\mathrm{N}$-cadherin $(14,16)$. But higher order unbinding events were also observed that point to additional mechanisms of interaction. These findings will be published in detail in a separate study. In the presence of EGTA-binding ( $5 \mathrm{mM})$, activity of Dsg1 transinteraction was strongly reduced to $15 \% \pm 8 \%$

\section{Figure 4}

Localization of Dsg1, Dsg3, and plakoglobin at cell-to-bead contacts. Dsg1-coated beads (A-D) and Fc-coated beads (E and $\mathbf{F})$ were immunostained following settlement on HaCaT cells for 30 minutes. Immunostaining for Dsg1 (B) showed localization of Dsg1 at the cell surface underneath most Dsg1-coated beads, which are visualized in A by corresponding phase contrast microscopic image. Note halo-like accumulation of cellular Dsg1 and plakoglobin around the bead attachment sites (arrows in B and D). Similarly, Dsg3 (C) was detected at cell-bead contacts. In contrast, no immunoreactivity for Dsg1 was found underneath $\mathrm{Fc}$-coated beads visualized in a corresponding phase contrast figure (E and F). Scale bar: $10 \mu \mathrm{m}$ for all panels $(n=5)$. of control values (Figure 7B). PF-IgGs 1 and 2 did not significantly reduce binding activity on the single molecule level $(35 \mu \mathrm{g} / \mathrm{ml}$, 30 minutes). Binding activity was $106 \% \pm 12 \%$ after treatment with PF1-IgGs and was even surprisingly higher following treatment with PF2-IgGs $(167 \% \pm 4 \%)$. To exclude the possibility that antibody-induced crosslinking of transinteracting Dsg1 ectodomains might have caused this increase in binding activity, we prepared antigen-binding fragments (Fabs) from the patients' IgG fractions. When applied at the same protein concentrations, PF1-IgG Fab fragments as well as PF2-IgG Fab fragments did not change Dsg 1 transinteraction $(94 \% \pm 11 \%$ and $107 \% \pm 9 \%$ of controls). This indicates that the increase in binding activity to $167 \%$ observed with PF2-IgGs was probably caused to some degree by antibody-mediated cross-bridging between Dsg1 molecules bound to plate and tip. In contrast to both PF-IgGs, the monoclonal antibody directed against the extracellular domain of Dsg1 (1:50) reduced binding activity to $42 \% \pm 9 \%$ of control values. Taken together, these experiments demonstrate that PF-IgGs did not block transinteraction of Dsg1 molecules in a cell-free system whereas PF-IgGs reduced Dsg1-mediated adhesion only under conditions in which they acted on keratinocytes.

\section{Discussion}

PF-IgGs reduce Dsg1-mediated adhesion by mechanisms other than steric bindrance. Our study demonstrates for what we believe is the first time at the single molecule level that Dsg1 molecules undergo homophilic, $\mathrm{Ca}^{2+}$-dependent interaction and provides further insight into the mechanisms involved in skin blistering induced by pemphigus autoantibodies. During the last decade, autoanti-
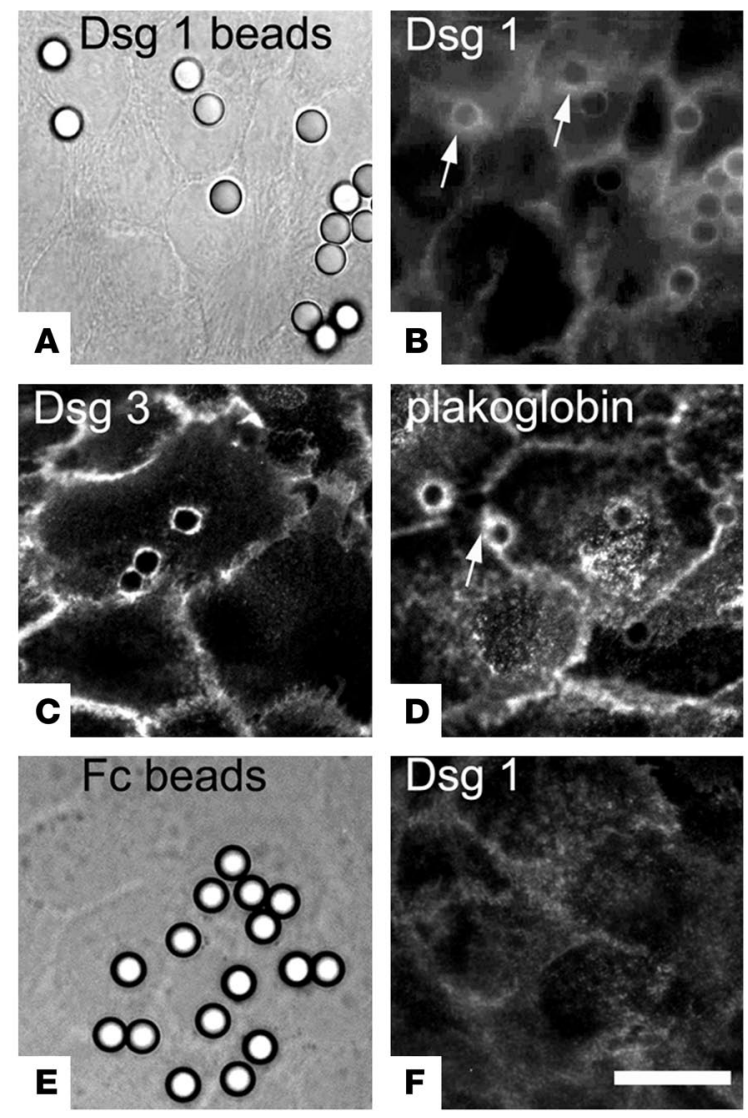

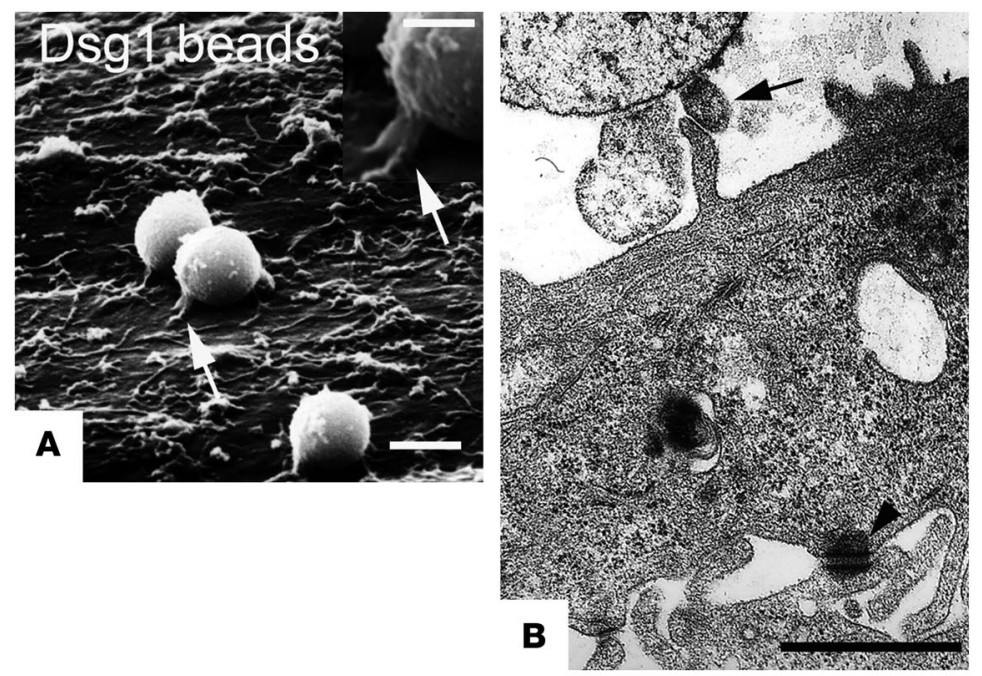

Figure 5

Characterization of cell-to-bead contacts induced by Dsg1-coated beads. Scanning electron microscopy $(\mathbf{A})$ and transmission electron microscopy (B) were used to characterize contacts between HaCaT cells and Dsg1-coated beads formed after 30 minutes of settlement. Finger-like processes were present underneath beads with direct contact to $\mathrm{HaCaT}$ cells (arrows). These processes were similar to the desmosome-bearing processes found at contact sites between neighboring cells (arrowhead). Scale bars: $2.5 \mu \mathrm{m}(\mathbf{A}), 1.25 \mu \mathrm{m}$ (A, inset); $1.0 \mu \mathrm{m}(\mathbf{B})$.

bodies against Dsgs have been identified as the main cause of blister formation in PF and PV $(1-4,22,23)$. Moreover, it is generally believed that Dsg1 is predominantly expressed in the superficial layers where little Dsg3 is present to compensate for the reduced function of Dsg1. This observation has been taken to explain why $\mathrm{PF}$ is characterized by superficial skin blistering whereas PV is associated with suprabasal blistering (8). However, the molecular mechanisms underlying skin blister formation induced by pemphigus antibodies are still unclear. Two hypotheses have been proposed as possible explanations for why scattering of keratinocytes is induced following binding of pemphigus antibodies (7, 9, 24-26). First, it is possible that binding of PF antibodies, which are known to be primarily directed against the first extracellular domain (27), would disrupt Dsg-mediated adhesion through steric hindrance. On the other hand, there are reports indicating

\section{Figure 6}

Effect of PF-IgGs on binding of Dsg1-coated beads to HaCaT cells. Beads were allowed to settle on the surface of HaCaT cells for 30 minutes (control). Number of bound beads was reduced by simultaneous incubation of EGTA ( $5 \mathrm{mM}, 30$ minutes). Incubation of monolayers with attached beads for an additional 30 minutes with IgG fractions from 2 patients (PF1- and PF2-lgG, $35 \mu \mathrm{g} / \mathrm{ml}$ each) as well as with a monoclonal antibody (1:50) directed against the extracellular domain of human Dsg1 significantly reduced the number of bound beads (label in white box and white bars). Immunoabsorption using Dsg1-coated beads but not control absorption using VE-cadherin-labeled beads completely abolished the effect of PF-IgGs on bead adhesion. Preincubation of $\mathrm{HaCaT}$ cells with PF-IgGs prior to bead settlement also reduced bead binding (label in gray box and gray bars) whereas preincubation of beads with PF-IgGs did not inhibit bead binding. In contrast, the monoclonal Dsg1 antibody reduced bead binding also when applied for preincubation with beads, indicating a different mechanism underlying the reduction of Dsg1 adhesion ( $n=6$ for each condition). that pemphigus antibodies induce several intracellular signaling events that may lead to decreased desmosomal adhesion (10-13). Recently, it has been demonstrated that PV-IgGs induce activation of p38 MAPK with subsequent phosphorylation of hsp 27 (28).

In our study, treatment of cultured keratinocytes with PF-IgGs induced intercellular gap formation and disappearance of Dsg1 from intercellular junctions. This was accompanied by significant reduction of Dsg1-mediated adhesion measured as number of surface-bound Dsg1coated beads resisting laser trapping. Immunodepletion of PF-IgGs using Dsg1-coated beads completely abolished these effects of PF-IgG, indicating that autoantibodies against Dsg1 were responsible for the biologic effects in our in vitro system and that the recombinant protein retained its native conformation during the purification procedure $(4,29)$.

However, in contrast to experiments using the monoclonal Dsg1 antibody, binding of beads to keratinocytes was not significantly reduced when Dsg1-coated beads were preincubated with PF-IgGs before bead settlement. These data indicate that PF-IgGs reduced Dsg1 binding by different, cell-dependent mechanisms whereas the monoclonal Dsg1 antibody seemed to directly inhibit Dsg1 adhesion. This was confirmed by studies using AFM in a cell-free system in which we demonstrated that the monoclonal antibody but not PF-IgGs inhibited Dsg1 transinteraction. The increased binding activity in the presence of PF2-IgGs was likely caused by antibody-mediated cross-linking between plate- and tip-bound Dsg1 ectodomains as indicated by experiments with Fab fragments prepared from PF2-IgG.

The novel result of our study is that PF-IgGs did not inhibit Dsg1 binding by steric hindrance but rather reduced Dsg1-mediated adhesion by antibody-mediated cellular responses. IgG fractions used in this study were taken from 2 patients afflicted with severe forms of PF. These IgG fractions also caused dissociation of keratinocytes in vitro under conditions where no steric hindrance of Dsg1 binding was detected, indicating that steric hindrance is not required for induction of the disease. However, since the goal of the study was not to determine the IgG epitope recognized by $\mathrm{PF}$ patient antibodies, we cannot exclude the possibility that IgG fractions that inhibit Dsg transinteraction may be present in other patients suffering from PF. But our experiments clearly

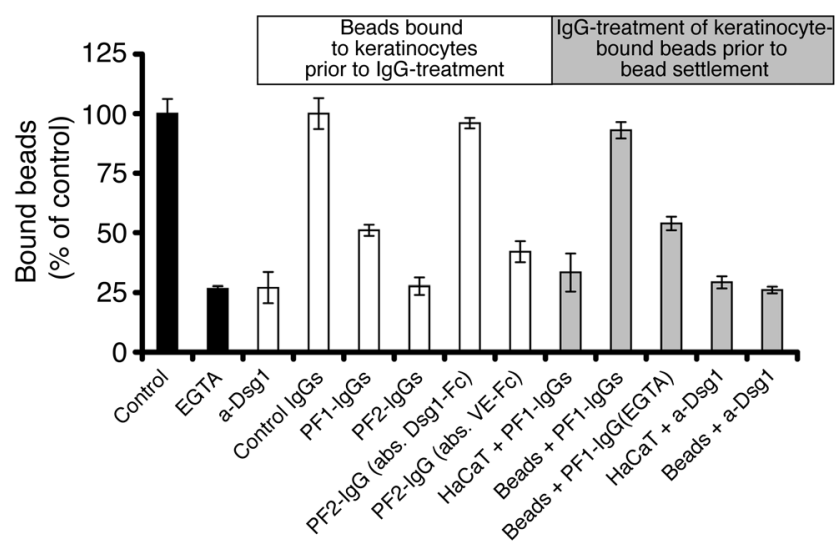


A
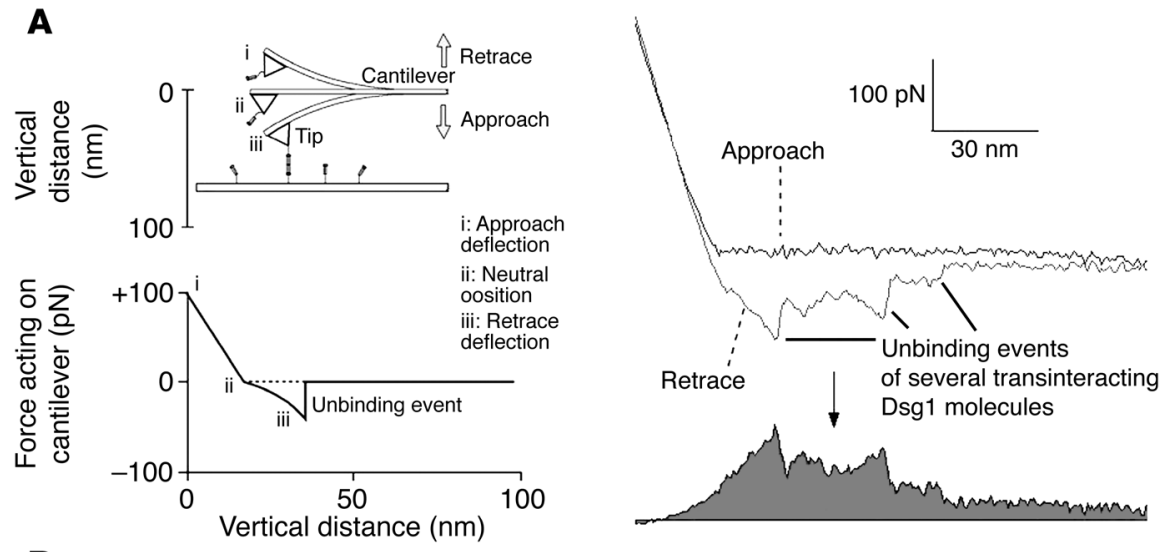

B

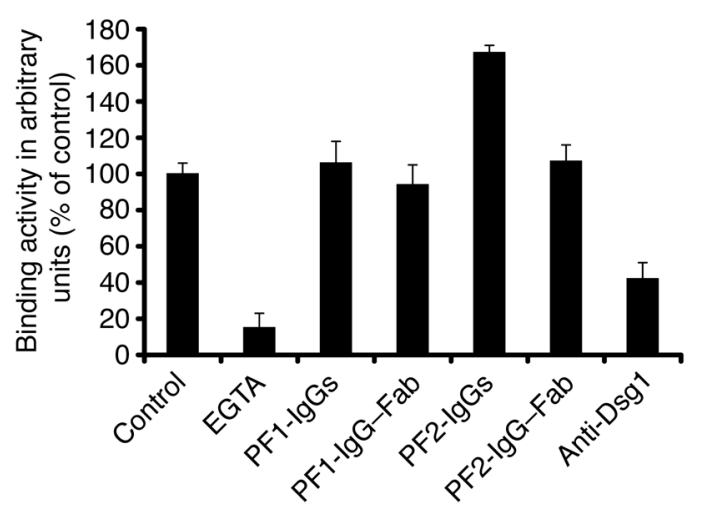

\section{Figure 7}

Binding activity of Dsg1 in the presence or absence of PF-IgGs probed by AFM. (A) General working principle of force-distance cycles. Dsg1-Fc is covalently attached by PEG-linkers to the plate and cantilever tip of the AFM setup. Molecules are brought into contact by downward movement of the tip. During upward movement, a downward deflection of the cantilever will occur if plateand tip-bound Dsg1 molecules undergo binding (left). Retrace and approach were subtracted, and the area below the resulting curve was integrated and taken as a measure for the average binding activity (right, gray). (B) Bar diagram shows binding activities of Dsg1 molecules in the presence or absence of antibodies. Binding activity was significantly reduced by incubation with EGTA ( $5 \mathrm{mM}, 30$ minutes) or the monoclonal antibody directed against Dsg1 (1:50). However, PF-lgGs (35 $\mu \mathrm{g} / \mathrm{ml}, 30 \mathrm{~min}$ ) did not reduce binding events in this cell-free system. Fab fragments from the PF-IgG fraction have been applied to rule out possible crosslinking effects of the PF-IgGs and were found not to reduce Dsg1-mediated binding $(n=4$ for each condition). show that steric hindrance is not a general mechanism by which skin blistering is mediated in PF patients, and we show further that an inhibitory mouse monoclonal antibody does not cause cellular dissociation of $\mathrm{HaCaT}$ cells.

PF-IgGs induced dissociation of keratinocytes and loss of Dsg1. In our study, incubation with PF-IgGs induced formation of numerous intercellular gaps in monolayers of $\mathrm{HaCaT}$ cells. Dissociation of keratinocytes in response to incubation with PF-IgGs was paralleled by profound loss of Dsg1 immunoreactivity. Whereas Dsg3 was still present in cellular processes spanning intercellular gaps, Dsg1 staining had largely disappeared in cell groups exhibiting gaps. These data are in line with previous studies that demonstrated that PV antibodies induced Dsg3 degradation $(10,11)$. Moreover, recent studies reported that PV-IgGs both reduced gene expression and increased phosphorylation of Dsg3 and other desmosomal plaque components. Expression of cytoskeletal proteins like cytokeratins was also found to be reduced following treatment with PV-IgGs (30). In this context, we believe our results are the first to demonstrate that PF-IgGs cause dissociation of cultured keratinocytes within a time course similar to that described previously for antibodies from PV patients (12). Also in favor of signaling events, other studies demonstrated that PV-IgGs caused activation of protein kinase $\mathrm{C}$ and required the expression of plakoglobin for cell dissociation $(7,12,25)$. Moreover, our experiments indicate that inhibition of Dsg 1 binding by steric hindrance is not sufficient to induce keratinocytes to detach. The monoclonal Dsg1 antibody reduced binding of Dsg1-coated beads as well as transinteraction of single Dsg1 molecules (AFM experiments) but was not sufficient to cause dissociation of keratinocytes. If cell dissociation in vitro reflects the skin blistering activity of PF-IgG, these results indicate that reduction of Dsg1-mediated adhesion by steric hindrance may not be sufficient for the pathogenesis of PF.

$\mathrm{Ca}^{2+}$-dependent homophilic transinteraction of Dsg1. Our results from AFM experiments clearly demonstrate that Dsg1 is capable of undergoing specific homophilic transinteraction in a $\mathrm{Ca}^{2+}$-dependent manner. In our AFM experiments, in which Dsg1 was coupled via flexible linkers to tip and plate of the AFM, binding was significantly reduced following incubation with either EGTA or the inhibitory monoclonal antibody directed against Dsg1.

The unbinding force of 2 transinteracting Dsg1 molecules was in the range of the forces measured by AFM for other members of the cadherin family that bind in homophilic manner, such as VE-cadherin $(35-55 \mathrm{pN})$ and $\mathrm{N}$-cadherin $(40 \mathrm{pN})(14,16)$. These data clearly demonstrate that Dsg1 is able to undergo homophilic transinteraction. However, it has to be emphasized that the in vitro conditions of our assay may not completely apply to the in vivo situation where members of both the Dsg and desmocollin (Dsc) subfamilies seemed to build heterophilic complexes. Heterophilic interaction has been shown by cell aggregation assays using cells expressing either Dsg1 or Dsc1 $(31,32)$. It has been proposed that the ratio between Dsg1 and Dsc1 is a critical determinant of desmosomal adhesion (33). Different studies using the first 2 extracellular domains of Dsg2 and Dsc2 reported both homophilic and heterophilic interaction of Dsg2 (34).

Taken together, our AFM experiments demonstrate that PFIgGs do not inhibit homophilic transinteraction of Dsg1 molecules by steric hindrance. However, it cannot completely be ruled out that heterophilic interactions of Dsg1 with Dsc1 may be more sensitive to PF IgG. Thus, further studies are needed 
to determine the contribution of homophilic versus heterophilic mechanisms of desmosomal adhesion and to characterize the underlying molecular binding properties.

\section{Methods}

Cell culture. The immortalized human keratinocyte cell line HaCaT (a kind gift from P. Boukamp, German Cancer Research Center (DKFZ), Heidelberg, Germany) was grown in DMEM (Invitrogen Corp.) supplemented with $50 \mathrm{U} / \mathrm{ml}$ penicillin-G, $50 \mu \mathrm{g}$ streptomycin, and $10 \% \mathrm{FCS}$ (Biochrom) in a humidified atmosphere (95\% air, $5 \% \mathrm{CO}_{2}$ ) at $37^{\circ} \mathrm{C}$. The cultures were used for all experiments when grown to confluent monolayers, and Dsg1 expression was detected by dot blot analysis (Figure 1) as well as immunostaining (day 7 after plating) (see Figure 3A). For dot blot analysis, cells from a T25 tissue flask were harvested in $150 \mu \mathrm{l}$ of HBSS following incubation for 20 minutes in PBS consisting of $137 \mathrm{mM} \mathrm{NaCl}, 2.7 \mathrm{mM} \mathrm{KCl}, 8.1 \mathrm{mMNa}_{2} \mathrm{HPO}_{4}$, and $1.5 \mathrm{mM} \mathrm{KH} 2 \mathrm{PO}$, pH 7.4) containing 1\% EGTA (Roth), followed by 10 minutes of incubation in EGTA/trypsin (0.025\%/0.05\% final concentration each). Dot blotting was performed as described below.

Cytochemistry. HaCaT cells were grown on cover slips to confluence as described above (7 days) and incubated with PF-IgGs or control IgGs for 24 hours at $37^{\circ} \mathrm{C}$. After incubation with PF-IgG, culture medium was removed and monolayers fixed for 10 minutes at room temperature (RT) with $2 \%$ formaldehyde (freshly prepared from paraformaldehyde) in PBS. Afterwards, monolayers were treated with $0.1 \%$ Triton X-100 in PBS for 5 minutes at RT. After rinsing with PBS at RT, HaCaT cells were preincubated for 30 minutes with $10 \%$ normal goat serum (NGS) and $1 \%$ BSA at RT and incubated for 16 hours at $4{ }^{\circ} \mathrm{C}$ with mouse monoclonal antibodies directed against the ectodomain of human Dsg1 (clone p124; Progen Industries Ltd.) or Dsg3 (Zytomed) (dilution 1:100 in PBS each). After several rinses with PBS (3 times for 5 minutes each time), monolayers were incubated for 60 minutes at RT with Cy3-labeled goat anti-mouse IgGs (Dianova). For visualization of F-actin, Alexa-phalloidin (MoBiTec; diluted 1:60 in PBS) was used (incubation for 1 hour at RT). Cells incubated with antibodies or Alexa-phalloidin were rinsed with PBS (3 times for 5 minutes each time). Cover slips were mounted on glass slides with $60 \%$ glycerol in PBS containing 1.5\% n-propyl gallate (Serva) as antifading compound. For visualization of Dsg1, Dsg3, and plakoglobin in association with bound Dsg1-coated beads, Dynabeads could not be used because of strong autofluorescence. Therefore, immunolocalization studies were performed with latex sulphate beads (Interfacial Dynamics Corp.). Bead solution ( $125 \mu \mathrm{l} ; 5 \mu \mathrm{l}$ packed beads) was washed twice in 2-morpholinoethane sulfonic acid (MES) (25 mM, pH 6.0) buffer by centrifugation and resuspension at $3000 \mathrm{~g}$ for 10 minutes. Beads were resuspended in $500 \mu \mathrm{l}$ MES including $2.5 \mu \mathrm{g}$ Dsg1-Fc and incubated overnight at RT under permanent slow overhead rotation. After centrifugation at $3000 \mathrm{~g}$ for $10 \mathrm{~min}$ utes, beads were washed 3 times in $1 \mathrm{ml}$ PBS containing $0.1 \%$ albumine. Beads were stored for up to 10 days under permanent overhead rotation at $4{ }^{\circ} \mathrm{C}$. After incubation of $\mathrm{HaCaT}$ with Dsg1-coated microbeads for 30 minutes, culture medium was removed and monolayers were treated as described above. Monolayers were examined using an LSM 510 (Zeiss). Images were processed using Adobe Photoshop 7.0 software.

Purification of PF-IgG. Sera from 2 PF patients whose diagnosis was confirmed clinically, histologically, and serologically and from a volunteer without any skin disease (control) were used for the present study. Subjects provided informed consent to the Department of Dermatology, University of Lübeck for the research studies. IgG fractions were purified by affinity chromatography using protein A agarose (Oncogene), eluted by citrate buffer (25 mM, pH 2.4), and immediately dialyzed against HBSS. The purity of isolated IgGs was checked by Coomassie blue staining of $7.5 \%$
SDS-PAGE. Activities against Dsg1 of IgGs purified from PF/control sera and purchased mouse monoclonal antibody directed against Dsg1 were adapted by dot blot immunodetection analysis of recombinant Dsg1-Fc (0.1-1 $\mu \mathrm{g}$ per dot) transferred to nitrocellulose using mouse monoclonal Dsg1 antibody followed by HRP-labeled goat anti-mouse IgGs (Dianova) as secondary antibody and the enhanced chemiluminescence technique (Amersham Biosciences) or biotinylated PF-IgGs and biotinylated control IgGs followed by HRP-labeled streptavidin (Dianova). For biotinylation, $300 \mu \mathrm{g}$ of PF-IgGs was incubated twice for 2 hours at RT with $20 \mu \mathrm{g} / \mu \mathrm{l}$ N-hydroxy-succimide-biotin (NHS-Biotin) (Pierce Biotechnology Inc.), and afterwards reaction was stopped using saturated glycin in PBS followed by dialysis against PBS overnight at $4^{\circ} \mathrm{C}$. Dot blotting of $\mathrm{HaCaT}$ cells was performed as indicated above using the monoclonal antibodies directed against Dsg1 and Dsg3, respectively.

Preparation of Fab fragments. PF-IgGs were dialyzed overnight against buffer A (0.1 M Tris, pH 8.0, 2 mM EDTA) at $4^{\circ} \mathrm{C}$. Papain (Sigma-Aldrich) was incubated in buffer A containing dithiothreitol (DTE) for 15 minutes at $37^{\circ} \mathrm{C}$ for activation and added at $1 \%$ final concentration to PF-IgG fractions for a 2 -hour incubation at $37^{\circ} \mathrm{C}$. Afterwards, reaction was stopped by incubation for 1 hour at $37^{\circ} \mathrm{C}$ in $2 \mathrm{mM}$ iodacetamid (35). Preparations of Fab fragments were analyzed by Coomassie blue staining of $7.5 \%$ SDSPAGE and by Western blotting using HRP-labeled goat anti-FC IgGs or goat anti-Fab IgGs (Dianova; 1:600 each) followed by HRP-labeled rabbit anti-goat IgGs as outlined above. Capability of Fab fragments to bind to recombinant Dsg1 was demonstrated by dot blot analysis.

Recombinant Dsg1-Fc. The transfer vector $\mathrm{pEVmodPFIg}$, containing the sequence of the entire extracellular domain (EC 1-5) of Dsg1 and the constant region of human IgG 1 (Fc), was a kind gift from Masayuki Amagai (Keio University School of Medicine, Tokyo, Japan) (4). For expression in eucaryotic cells, the Dsg1-Fc sequence was excised from pEVmodPFIg by digestion with BglII and KpnI and ligated with similarly cut pEGFP-N3 mammalian expression vector (BD Biosciences - Clontech). Dsg1-Fc and VE-cadherin were expressed by stably transfected $\mathrm{CHO}$ cells and purified from culture supernatants by affinity chromatography using protein A agarose (Oncogene). The protein was eluted by citrate buffer $(25 \mathrm{mM}, \mathrm{pH} 2.4)$ and immediately dialyzed against HBSS for 16 hours at $4^{\circ} \mathrm{C}$ and stored in aliquots at $-80^{\circ} \mathrm{C}$. Protein content was determined using the Bradford method, and purity was checked by Coomassie blue staining of $10 \%$ SDS-PAGE. Western blotting and immunodetection were performed as described above using mouse monoclonal antibody against Dsg1 as well as goat anti-human IgGs directed against the Fc-portion of the fusion protein. Both antibodies detected the recombinant Dsg1-Fc fusion protein as a single band migrating slightly above the calculated molecular weight (not shown).

Coating of polystyrene beads. After vortexing, $10 \mu \mathrm{l}$ solution of protein Acoated superparamagnetic polystyrene microbeads (Dynabeads, diameter $2.8 \mu \mathrm{m}$; Dynal) containing $2 \times 10^{9}$ beads $/ \mathrm{ml}$ were washed 3 times using $100 \mu \mathrm{l}$ of buffer A (100 mM sodium phosphate buffer, $\mathrm{pH}$ 8.1). Washing was performed by immobilization of beads for 1 minute in a magnetic tube holder (MPC-E-1; Dynal Biotech) and reuptake in the corresponding buffer. Washed beads were suspended in $100 \mu \mathrm{l}$ of $100 \mathrm{mM}$ sodium phosphate buffer, $\mathrm{pH}$ 8.1, in HBSS containing $10 \mu \mathrm{g}$ of Dsg1-Fc or of the Fc part of human IgGs (Dianova) and allowed to react for 16 hours at $4^{\circ} \mathrm{C}$ under permanent slow overhead rotation to avoid aggregation. After washing 3 times for 5 minutes each time in $100 \mu \mathrm{l}$ of buffer $\mathrm{A}$ and 3 times for 5 minutes each time in buffer B (100 mM sodium borate, $\mathrm{pH} 9.0)$, beads were incubated for 45 minutes at RT in $100 \mu \mathrm{l}$ buffer B containing $0.54 \mathrm{mg}$ dimethyl pimelimidate dihydrochloride (DMP) (Pierce Biotechnology Inc.) to covalently cross-link protein A and bound Fc parts. After washing 2 times for 5 minutes each time in buffer C (100 $\mu 10.2 \mathrm{M}$ 
ethanolamine, $\mathrm{pH}$ 8.0), beads were incubated in buffer $\mathrm{C}$ for 2 hours at RT. Finally, beads were washed 3 times for 5 minutes each time in HBSS and stored in HBSS at $4{ }^{\circ} \mathrm{C}$ for up to 8 days under permanent slow overhead rotation to avoid aggregation of beads. The concentration of beads in these stocks was about $1.6 \times 10^{8}$ beads $/ \mathrm{ml}$.

Immunoabsorption experiments. PF-IgGs were added to $1 \mathrm{ml}$ of DMEM (35 $\mu \mathrm{g} / \mathrm{ml}$ final concentration) and incubated overnight at $4^{\circ} \mathrm{C}$ with Dynabeads coated with $15 \mu \mathrm{g}$ recombinant Dsg1-Fc or VE-cadherin-Fc as described above. To saturate potential free protein A binding sites, beads were postincubated with $200 \mu \mathrm{l}$ of human control $\operatorname{IgGs}(0.3 \mu \mathrm{g} / \mathrm{ml})$ for 1 hour at RT. Following immunoabsorption, beads were separated using a magnetic tube holder, and DMEM containing the IgG fractions was applied to HaCaT monolayers.

Laser tweezer. The setup was used as described previously (17). The home-built laser tweezer setup consisted of an Nd:Yag laser (1064 nm), the beam of which was expanded to fill the back aperture at a high NAobjective $(100 \times 1.3$ oil; Zeiss), coupled through the epi-illumination port of an Axiovert 135 microscope (Zeiss) and reflected to the objective by a dicroic mirror (FT 510; Zeiss). Through all experiments, the laser intensity was $42 \mathrm{~mW}$ in the focal plane. Coated beads ( $10 \mu \mathrm{l}$ of stock solution) were suspended in $200 \mu \mathrm{l}$ of culture medium and allowed to interact with $\mathrm{HaCaT}$ monolayers for 30 minutes at $37^{\circ} \mathrm{C}$ before measuring the number of bound beads (control values). Beads were considered tightly bound when resisting laser displacement at the $42-\mathrm{mW}$ setting. For every condition, 100 beads were counted. Afterwards, control and PF-IgG fractions $(35 \mu \mathrm{g} / \mathrm{ml})$ were applied for 30 minutes, and the number of bound beads was counted again. Percentage of beads resisting laser displacement under various experimental conditions was normalized to control values. For preincubation experiments, either $\mathrm{HaCaT}$ cells or Dsg1-coated beads were incubated with PF-IgGs ( $35 \mu \mathrm{g} / \mathrm{ml}, 30$ minutes) and rinsed twice in HBSS before beads were allowed to settle on $\mathrm{HaCaT}$ cells for 30 minutes. Negative controls were performed using Dsg1-coated beads incubated on the surface of HaCaT cells in the presence of $5 \mathrm{mM}$ EGTA (Roth) or in the presence of monoclonal mouse IgG antibody (1:50 in HBSS) directed against Dsg-1 (clone p124; Progen Industries Ltd.).

Scanning electron microscopy. HaCaT monolayers with adhering Dsg1coated Dynabeads were fixed overnight with $6.5 \%$ glutaraldehyde in HBSS. After dehydration with graded acetone series, critical point drying, and sputtercoating with palladium-gold (CPD 030, BAL-TEC), cells were examined with a JSM-840 scanning electron microscope (JEOL).

Transmission electron microscopy. $\mathrm{HaCaT}$ monolayers with adhering Dsg1-coated Dynabeads were fixed in $2.5 \%$ glutaraldehyde containing $0.01 \%$ ruthenium red in $0.1 \mathrm{M}$ sodium cacodylate, $\mathrm{pH} 7.35$, for 1 hour at $4{ }^{\circ} \mathrm{C}$. After rinsing in $0.1 \mathrm{M}$ cacodylate ( 3 times for 5 minutes each time), cells were postfixed in $2 \% \mathrm{OSO}_{4}$ in $0.1 \mathrm{M}$ cacodylate for 1 hour at RT. Cells were rinsed again in cacodylate and dehydrated in ascending concentrations of methanol $(25 \%, 50 \%, 70 \%, 80 \%, 95 \%$ [twice], $100 \%$ [twice]; 10 minutes each time). Following incubation in 70\% methanol, monolayers were placed for 1 hour in saturated uranyl acetate in $70 \%$ methanol for 1 hour in the dark. After dehydration, cells were equilibrated in propylene oxide ( 2 times for 10 minutes each time) and embedded in Epon 812, semithin sections $(1 \mu \mathrm{M})$ were stained with toluidine blue, and ultrathin sections were contrasted with uranyl acetate and lead citrate and examined with a LEO AB 912 electron microscope.

Atomic force microscopy measurements. Dsg1-Dsg1 interactions were characterized by force-distance measurements of Dsg1 coupled via flexible linkers to the tip and substrate of a Bioscope AFM driven by a Nanoscope III controller (Digital Instruments Inc.). Dsg1 was linked covalently to the Si3N4 tip of the cantilever (Park Scientific Ltd.) and freshly cleaved mica plates (Wacker) using PEG spacers containing an amino-reactive cross-linker group (NHS ester) at 1 end and a thiol-reactive group (2-[pyridyldithio] propionate) at the other, as previously described in detail (21). The NHS group served to link PEG to free amino acid groups at both the Si3N4 tip and the $\mathrm{SiOH}$ plate introduced by treatment of the tip and plate with 2 -aminoethanol $\mathrm{HCl}$ (Sigma-Aldrich). Binding events were measured in HBSS by force-distance cycles at amplitudes of $300 \mathrm{~nm}$ and at frequencies ranging from $1-2 \mathrm{~Hz}$. Force-distance cycles were performed either at constant lateral positions or with lateral shifts of $1 \mathrm{~nm} / \mathrm{s}$. Force-distance cycles were analyzed as described previously in detail $(15,16)$.

Statistics. Differences in bead adhesion or single molecule transinteraction between different protocols were assessed using 2-tailed Student's $t$ test. Values throughout are expressed as mean \pm SEM. Statistical significance is assumed at $P<0.05$.

\section{Acknowledgments}

We are grateful to Masayuki Amagai (Keio University School of Medicine, Tokyo, Japan) for supplying us with the Dsg1-Fc construct and helpful discussion and to P. Boukamp (German Cancer Research Center (DKFZ), Heidelberg, Germany) for supplying the $\mathrm{HaCaT}$ cell line. We thank Gabriele Königer and Siglinde Schenk for excellent technical assistance. These studies were supported by a grant from the Deutsche Forschungsgemeinschaft (SFB 487, TP B5).

Received for publication September 27, 2004, and accepted in revised form July 26, 2005.

Address correspondence to: Detlev Drenckhahn, Institute of Anatomy and Cell Biology, Julius-Maximilians-University, Koellikerstrasse 6, D-97070 Würzburg, Germany. Phone: 49-931-31-2702; Fax: 49-931-31-2712; E-mail: anat015@mail.uni-wuerzburg.de.

Jens Waschke and Paola Bruggeman contributed equally to this work.
1. Stanley, J.R., Yaar, M., Hawley-Nelson, P., and Katz, S.I. 1982. Pemphigus antibodies identify a cell surface glycoprotein synthesized by human and mouse keratinocytes. J. Clin. Invest. 70:281-288.

2. Stanley, J.R., Koulu, L., and Thivolet, C. 1984. Distinction between epidermal antigens binding pemphigus vulgaris and pemphigus foliaceus autoantibodies. J. Clin. Invest. 74:313-320.

3. Amagai, M., Karpati, S., Prussick, R., Klaus-Kovtun, V., and Stanley, J.R. 1992. Autoantibodies against the amino-terminal cadherin-like binding domain of pemphigus vulgaris antigen are pathogenic. J. Clin. Invest. 90:919-926.

4. Amagai, M., Hashimoto, T., Green, K.J., Shimizu, N., and Nishikawa, T. 1995. Antigen-specific immunoadsorption of pathogenic autoantibodies in pem- phigus foliaceus. J. Invest. Dermatol. 104:895-901.

5. Nollet, F., Kools, P., and van Roy, F. 2000. Phylogenetic analysis of the cadherin superfamily allows identification of six major subfamilies besides several solitary members. J. Mol. Biol. 299:551-572.

6. Garrod, D.R., Merritt, A.J., and Nie, Z. 2002. Desmosomal cadherins. Curr. Opin. Cell Biol. 14:537-545.

7. Amagai, M. 2003. Desmoglein as a target in autoimmunity and infection. J. Am. Acad. Dermatol. 48:244-252.

8. Hashimoto, T. 2003. Recent advances in the study of the pathophysiology of pemphigus. Arch. Dermatol. Res. 295(Suppl. 1):S2-S11.

9. Kitajima, Y., Aoyama, Y., and Seishima, M. 1999. Transmembrane signaling for adhesive regulation of desmosomes and hemidesmosomes, and for cell- cell datachment induced by pemphigus IgG in cultured keratinocytes: involvement of protein kinase C. J. Investig. Dermatol. Symp. Proc. 4:137-144.

10. Aoyama, Y., and Kitajima, Y. 1999. Pemphigus vulgaris-IgG causes a rapid depletion of desmoglein 3 (Dsg3) from the Triton X-100 soluble pools, leading to the formation of Dsg3-depleted desmosomes in a human squamous carcinoma cell line, DJM-1 cells. J. Invest. Dermatol. 112:67-71.

11. Aoyama, Y., Owada, M.K., and Kitajima, Y. 1999. A pathogenic autoantibody, pemphigus vulgarisIgG, induces phosphorylation of desmoglein 3, and its dissociation from plakoglobin in cultured keratinocytes. Eur. J. Immunol. 29:2233-2240.

12. Caldelari, R., et al. 2001. A central role for the armadillo protein plakoglobin in the autoimmune disease 
pemphigus vulgaris. J. Cell Biol. 153:823-834.

13. Lo Muzio, L., et al. 2001. A possible role of catenin dyslocalization in pemphigus vulgaris pathogenesis J. Cutan. Pathol. 28:460-469.

14. Baumgartner, W., et al. 2000. Cadherin interaction probed by atomic force microscopy. Proc. Natl. Acad. Sci. U. S. A. 97:4005-4010.

15. Baumgartner, W., and Drenckhahn, D. 2002. Plasmalemmal concentration and affinity of mouse vascular endothelial cadherin, VE-cadherin. Eur Biophys. J. 31:532-538.

16. Baumgartner, W., Golenhofen, N., Grundhofer, N., Wiegand, J., and Drenckhahn, D. 2003. Ca2+ dependency of $\mathrm{N}$-cadherin function probed by laser tweezer and atomic force microscopy. J. Neurosci. 23:11008-11014

17. Baumgartner, W., Schutz, G.J., Wiegand, J., Golenhofen, N., and Drenckhahn, D. 2003. Cadherin function probed by laser tweezer and single molecule fluorescence in vascular endothelial cells. J. Cell Sci. 116:1001-1011.

18. Waschke, J., et al. 2004. Requirement of Rac activity for maintenance of capillary endothelial barrier properties. Am. J. Physiol. Heart. Circ. Physiol. 286:H394-H401.

19. Waschke, J., Drenckhahn, D., Adamson, R.H., and Curry, F.E. 2004. The role of adhesion and contraction in Rac 1-regulated endothelial barrier function in vivo and in vitro. Am. J. Physiol. Heart. Circ. Physiol. 287:H704-H711.
20. Windoffer, R., Borchert-Stuhltrager, M., and Leube, R.E. 2002. Desmosomes: interconnected calciumdependent structures of remarkable stability with significant integral membrane protein turnover. J. Cell Sci. 115:1717-1732.

21. Hinterdorfer, P., Baumgartner, W., Gruber, H.J., Schilcher, K., and Schindler, H. 1996. Detection and localization of individual antibody-antigen recognition events by atomic force microscopy. Proc. Natl. Acad. Sci. U. S. A. 93:3477-3481.

22. Amagai, M., Hashimoto, T., Shimizu, N., and Nishikawa, T. 1994. Absorption of pathogenic autoantibodies by the extracellular domain of pemphigus vulgaris antigen (Dsg3) produced by baculovirus. J. Clin. Invest. 94:59-67.

23. Zillikens, D., et al. 2001. Antibodies to desmogleins 1 and 3, but not to BP180, induce blisters in human skin grafted onto SCID mice. J. Pathol. 193:117-124.

24. Martel, P., and Joly, P. 2001. Pemphigus: autoimmune diseases of keratinocyte's adhesion molecules. Clin. Dermatol. 19:662-674.

25. Kitajima, Y. 2002. Mechanisms of desmosome assembly and disassembly. Clin. Exp. Dermatol. 27:684-690.

26. Whittock, N.V., and Bower, C. 2003. Targetting of desmoglein 1 in inherited and acquired skin diseases. Clin. Exp. Dermatol. 28:410-415.

27. Sekiguchi, M., et al. 2001. Dominant autoimmune epitopes recognized by pemphigus antibodies map to the $\mathrm{N}$-terminal adhesive region of desmogleins. J. Immunol. 167:5439-5448.

28. Berkowitz, P., et al. 2005. Desmosome signaling. Inhibition of p38MAPK prevents pemphigus vulgaris IgG induced cytoskeleton reorganisation. J. Biol. Chem. 280:23778-23784.

29. Hanakawa, Y., et al. 2003. Calcium-dependent conformation of desmoglein 1 is required for its cleavage by exfoliative toxin. J. Invest. Dermatol. 121:383-389.

30. Nguyen, V.T., et al. 2004. Pemphigus vulgaris IgG and methylprednisolone exhibit reciprocal effects on keratinocytes. J. Biol. Chem. 279:2135-2146.

31. Marcozzi, C., Burdett, I.D.J., Buxton, R.S., and Magee, A.I. 1998. Coexpression of both types of desmosomal cadherin and plakoglobin confers strong intercellular adhesion. J. Cell Sci. 111:495-509.

32. Tselepis, C., Chidgey, M., North, A., and Garrod, D. 1998. Desmosomal adhesion inhibits invasive behavior. Proc. Natl. Acad. Sci. U. S. A. 95:8064-8069.

33. Getsios, S., et al. 2004. Coordinated expression of desmoglein 1 and desmocollin 1 regulated intercellular adhesion. Differentiation. 72:419-433.

34. Syed, S.E., et al. 2002. Molecular interactions between desmosomal cadherins. Biochem. J. 362:317-327.

35. Rousseaux, J., Rousseaux-Prevost, R., and Bazin, H. 1983. Optimal conditions for the preparation of $\mathrm{Fab}$ and $\mathrm{F}\left(\mathrm{ab}^{\prime}\right) 2$ fragments from monoclonal IgG of different rat IgG subclasses. J. Immunol. Methods. 64:141-146. 